\title{
The Effects of the Solvents on the Macrocyclic Structures: From Rigid Pillararene to Flexible Crown Ether
}

\author{
Shuangshuang Wang, ${ }^{\oplus a}$ Yanzhen Yin, ${ }^{a}$ Jian Gao, ${ }^{, a}{ }^{a}$ Xingtang Liang $^{a}$ and Haixin Shi ${ }^{a}$ \\ ${ }^{a}$ Guangxi Colleges and Universities Key Laboratory of Beibu Gulf Oil and Natural Gas Resource \\ Effective Utilization, College of Petroleum and Chemical Engineering, Beibu Gulf University, \\ Qinzhou 535011, China
}

\begin{abstract}
The differences in the macrocyclic structures lead to different flexibilities, and yet the effect of solvents on the conformations is not clear so far. In this work, the conformations of four representational macrocyclic molecules (pillar[5]arene, $p$-tert-butyl calix[6]arene, benzylic amide macrocycle and dibenzo-18-crown-6) in three solvents with distinct polarity have been studied by all-atom molecular dynamics simulations. The structural features of the macrocycles in the solvents indicate that the conformations are related to the polarity of the solvents and the formation of hydrogen bonds. For the pillar[5]arene, the benzylic amide macrocycle and the dibenzo-18crown-6, that cannot form intramolecular hydrogen bonds, the polarity of solvents is the major contributing factor in the conformations. The formation of intramolecular hydrogen bonds, in contrast, determinates the conformations of the calix[6]arene. Furthermore, the slight fluctuations of the structures will result in tremendous change of the intramolecular hydrogen bonds of the macrocycles and the intermolecular hydrogen bonds between the macrocycles and the solvents. The current theoretical studies that serve as a basis for the macrocyclic chemistry are valuable for the efficient structural design of the macrocyclic molecules.
\end{abstract}

Keywords: macrocycles, molecular dynamics simulations, solvents, hydrogen bonds

\section{Introduction}

Since crown ether was discovered by C. J. Pedersen ${ }^{1,2}$ in 1967, lots of researches of macrocyclic chemistry have made rapid progress on it. During the subsequent decades, a large number of artificial macrocyclic compounds, e.g., calix $[n]$ arenes, ${ }^{3,4}$ pillar $[n] \operatorname{arenes}^{5-7}$ and benzylic amide rings, ${ }^{8,9}$ have been synthesized, giving rise to a considerable increase in research activity into all aspects of macrocyclic chemistry, especially in relation to molecular recognition and self-assembly processes. ${ }^{10}$ From a historic point of view, macrocyclic molecules have had an enormous impact on the fields of chemistry, ${ }^{11}$ biology, ${ }^{12}$ and medicine. ${ }^{13-15}$

The architectures of these macrocyclic molecules have ordained that they are of varying degrees of flexibility and ability of forming hydrogen bonds (H-bonds). The structures of several macrocyclic molecules are shown in Scheme 1. These selected macrocyclic molecules have certain representativeness. For example, ordinarily, the pillar[5]arenes $(\mathrm{P}[5] \mathrm{s})$ show unique pillar architectures and

*e-mail: jgao12@163.com are highly rigid compared with the p-tert-butyl calix[6] arenes $(\mathrm{C}[6] \mathrm{s})$, which are cup-shaped cyclic oligomers. It is reported that the large flexibility of the $C[6]$ s could lead to the easy inversion of their phenolic units. ${ }^{16,17}$ The benzylic amide macrocycles (BA rings) have four benzene rings linked to each other by a short chain formed by three atoms. In some cases, it could undergo a chair-to-boat conformational transition. ${ }^{18-20}$ Among all the macrocyclic molecules, the dibenzo-18-crown-6 types (D18C6), which are two benzene rings linked by polyethoxy chains, are of the most flexibility ${ }^{9,21}$ and could adopt numerous diamondlattice conformations. ${ }^{22}$ Therefore, generally speaking, the more flexible the molecule is, the more conformations it has. However, is it in line with the rule in any case? The relation of the structures of these molecules to solvents is not clear yet. In other words, how do the solvents affect their structures? How is the structural stability in the solvents? Is the stability of the structures related to the polarity of the solvents?

To shed light on these questions cogently, four models with several representative macrocyclic molecules (see Scheme 1) were built, and the conformations in three 


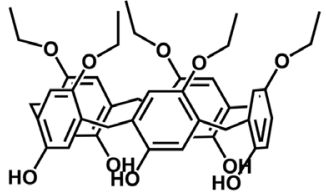

(a)

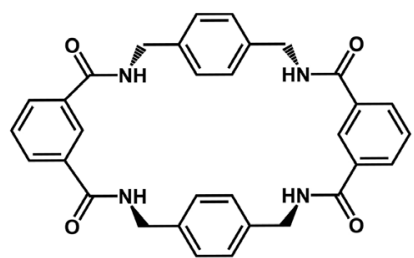

(c)

Scheme 1. The chemical structures of the macrocyclic molecules. (a), (b), (c), (d) are the P[5], the C[6], the BA ring and the D18C6, respectively.

solvents (chloroform $\left(\mathrm{CHCl}_{3}\right)$, dimethyl sulfoxide (DMSO) and water) were investigated by all-atom molecular dynamics (MD) simulations. The following detailed analysis explained the effect of polarity of the solvents on the structures of macrocycles. The influence of intramolecular and intermolecular $\mathrm{H}$-bonds on the structures of the macrocycles was also studied. In conclusion, the current studies allow us to systematically understand the mechanism of how the polarity of solvents affects macrocyclic structures, and are helpful to understand the host-guest matching.

\section{Methodology}

\section{Molecular models}

Scheme 1 shows the chemical structures of the four macrocyclic molecules. The initial coordinates of the four macrocyclic molecules, the $\mathrm{P}[5]$, the $\mathrm{C}[6]$, the $\mathrm{BA}$ ring and the D18C6, were extracted from the available threedimensional crystal structures of references 5, 23, 24, and 25 , respectively. The four molecules were constructed and energy-minimized, and then immersed independently in three cubic solvent boxes using the solvate module of the visualization program $\mathrm{VMD},{ }^{26}$ with a headspace of at least $30 \AA$ from each edge of the box to any atom of the macrocyclic molecules. The three cubic solvent boxes contain $1200 \mathrm{CHCl}_{3}, 1000$ DMSO and 2000 water, respectively. A $50 \mathrm{~ns}$ equilibrium simulation was performed for each system, and the trajectories were saved for later analysis.

\section{Molecular dynamics simulations}

All-atom MD simulations were employed to investigate the structural changes of macrocyclic molecules in the solvents. Langevin dynamics and the Langevin piston method were applied to maintain the temperature of $298 \mathrm{~K}$ and the pressure of $1 \mathrm{~atm} .{ }^{27}$ All simulations were performed using the parallel, scalable MD program NAMD 2.12 28 with the CHARMM 36 general force field (CGenFF) ${ }^{29}$ and the TIP3P water model. ${ }^{30}$ The rigid model of Dietz and Heinzinger (DH model), ${ }^{31}$ which had been merged into the CHARMM general force field, was used for $\mathrm{CHCl}_{3}$. The parameters for DMSO were taken from reference 32. The charge distributions of the fragments of the four macrocyclic molecules and the solvent molecules (water, $\mathrm{CHCl}_{3}$ and DMSO) were all provided in "The charge distributions used for the all-atom MD simulations" subsection of the Supplementary Information (SI) section. Covalent bonds involving hydrogen atoms were constrained to their equilibrium length employing the SHAKE/ RATTLE algorithms. ${ }^{33,34}$ The r-RESPA multiple time step algorithm $^{35}$ was employed to integrate the equations of motion with a time step of 2 and 4 fs for short- and longrange interactions, respectively. The short-range van der Waals and the electrostatic interactions were truncated at a smoothed $12.0 \AA$ spherical cutoff, and the long-range electrostatic force was evaluated by means of the Particle Mesh Ewald method. ${ }^{36}$ Visualization and analysis of the MD trajectories were performed with the VMD program. ${ }^{26}$

\section{Results and Discussion}

The molecular structures of macrocycles in solvents

For investigating the relations of the structures of these macrocyclic molecules to solvents, the structural changes of macrocyclic molecules in three solvents with different polarity, i.e., $\mathrm{CHCl}_{3}$, DMSO, water, were analyzed.

The $\mathrm{P}[5]$

The $\mathrm{P}[5]$, which is composed of hydroquinone rings linked by methylene bridges at para-positions, is of a symmetric structure with an overall pillar-like shape. Though the structure is relatively rigid, the benzene rings could swing slightly owning to the methylene bridges, which could lead to small conformational alterations of the $\mathrm{P}[5]$. The evolution of the angles between the five benzene rings and the plane composed of the five methylene carbon along with the time are examined. As the fluctuations of the five curves in a solvent are very similar, only one of the curves is shown in Figure 1. A cursory glance, the angles are about $90^{\circ}$ on average. However, the fluctuations in three solvents are different. The fluctuation range in water is greatest with $45^{\circ}$ to $120^{\circ}$ among the three solvents, while that in $\mathrm{CHCl}_{3}$ is minimal with $55^{\circ}$ to $110^{\circ}$. This is consistent 

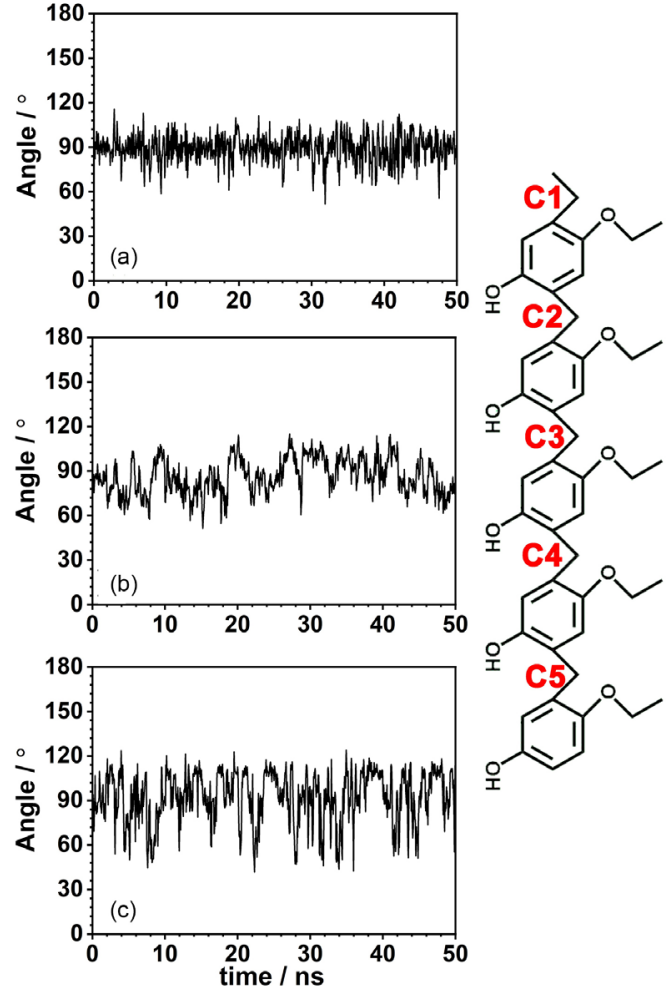

Figure 1. Evolution of the angles between one of the five benzene rings and the plane composed of the three methylene carbon atoms $(\mathrm{C} 1, \mathrm{C} 3$, C5) along with the increasing simulation time in (a) $\mathrm{CHCl}_{3}$, (b) DMSO and (c) water. with the order of the polarity of $\mathrm{CHCl}_{3}$, DMSO and water from low to high. Therefore, higher polarity of the solvents results in the bigger fluctuations of the structure.

To investigate the physical origin of these observations, the electrostatic interactions of the solvents with the five benzene rings (sol-ben-elec), with five $\mathrm{O} 1$ atoms (sol-O1-elec) and five $\mathrm{O} 2$ atoms (sol-O2-elec) are shown in Figure 2. According to the results, it can be seen that, regardless of the solvents, the sol-O1-elec interactions are strongest. In other words, the hydroxyl groups are the main cause of the electrostatic interactions. Furthermore, in DMSO and water, the intensity of the sol-O1-elec interactions is much higher than that in $\mathrm{CHCl}_{3}$. This further confirms that the higher polarity of the solvents results in the stronger electrostatic interactions of the solvents with the $\mathrm{P}[5]$, thus leading to the greater structural fluctuations. The representative structure of the $\mathrm{P}[5]$ is shown in Figure 3.

\section{The $\mathrm{C}[6]$}

The C[6], which is composed of phenol rings linked by methylene bridges at meta-positions, is of an asymmetric structure with an cup-like shape. The top-heavy structure makes it more flexible and prone to undergo conformational changes. The evolution of the angles between the six
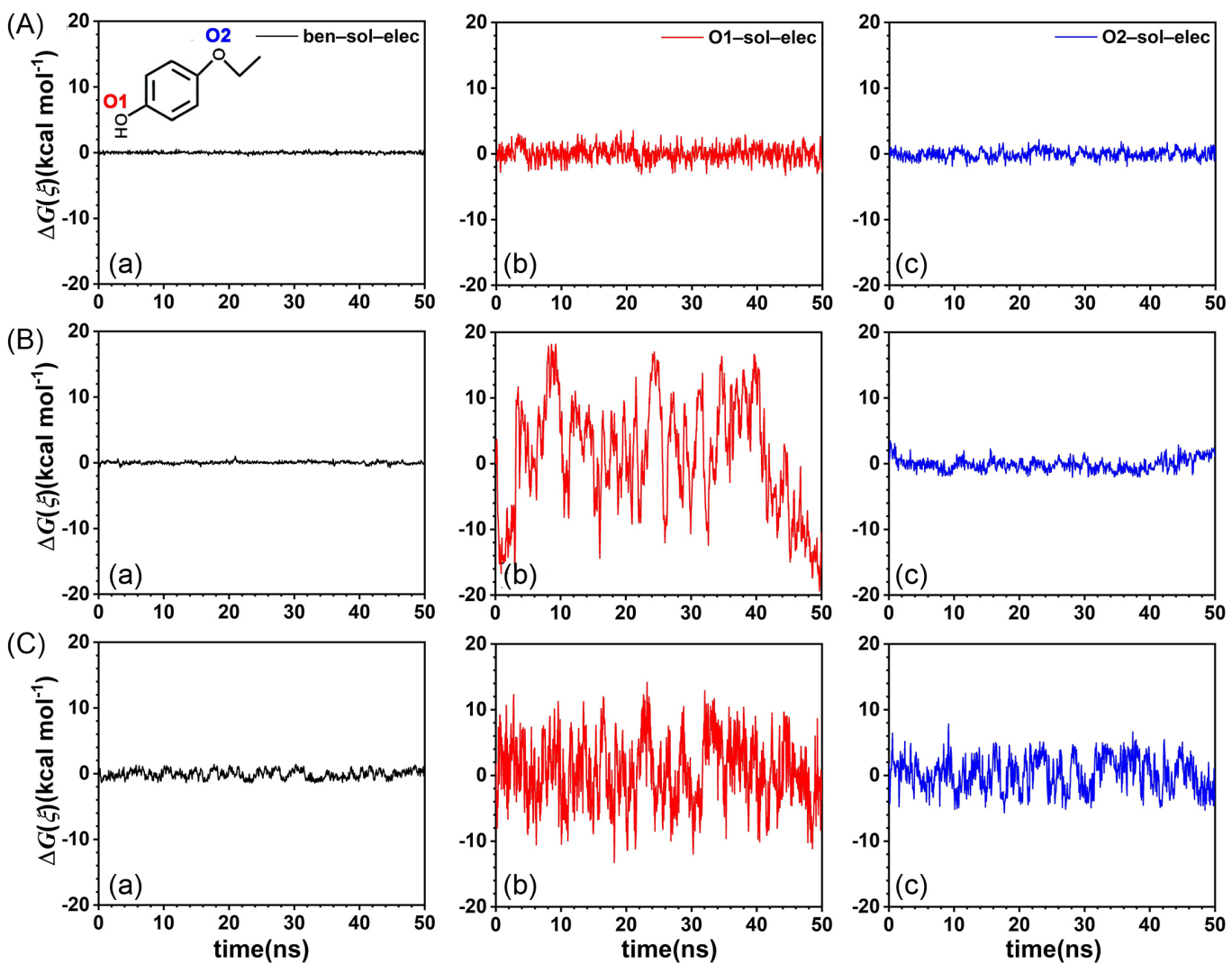

Figure 2. Evolution of electrostatic interactions of the solvent with (a) the five benzenes (ben-sol-elec), (b) five O1 atoms (O1-sol-elec) and (c) five O2 atoms $\left(\mathrm{O} 2-\right.$ sol-elec) of the $\mathrm{P}[5]$ along with the time in (A) $\mathrm{CHCl}_{3}$, (B) DMSO and (C) water. 


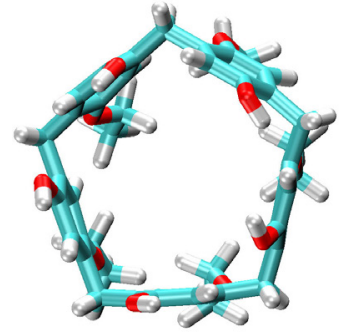

the primary rim

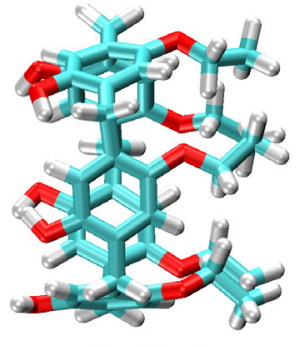

the flank
Figure 3. Snapshots of the representative structure of the P[5] from the primary rim and the flank.

benzene rings and the plane composed of the six methylene carbon atoms along with the time is shown in Figure 4. Unexpectedly, the fluctuations of the angles are smaller than that of the $\mathrm{P}[5]$, and those of the six benzene rings are inconsistent with each other.

By and large, the swing of the benzene rings of the $\mathrm{C}[6]$ is relatively slight and has not experienced sharp break in three solvents, and what's more, there are at least two benzene rings (for example ring 1 and 4) of which the swing amplitude is smaller than other rings in a solvent.
It indicates that the $\mathrm{C}[6]$ s can maintain a relatively stable conformation in the three solvents. It is also proved by analyzing the trajectories.

In $\mathrm{CHCl}_{3}$, the angles of the rings $(1,2,3,4,5,6)$ are about $40,90,100,135,40,150^{\circ}$, respectively. The representative structure of the $\mathrm{C}[6]$ in $\mathrm{CHCl}_{3}$ is shown in Figure 5. The structure resembles the shape of a chipped bowl. The rings $(1,4)$ extend outward, and particularly the methylene carbon atom between the ring 2 and 3 folds inward while the other five methylene carbons fold outward. The inward methylene carbon makes the benzene rings $(5,6)$ to have little sway. Therefore, the rings $(1,4,5,6)$ swing more slightly than the rings $(2,3)$. This structure is favorable to the six rings of the structure B forming a group and the six hydroxyl groups of the secondary rim forming $\mathrm{H}$-bonds end-to-end (see Figure 5). In DMSO and water, the fluctuations of the six rings are similar in a solvent, which are tiny and uniform. It indicates that the $\mathrm{C}[6] \mathrm{s}$ can maintain a relatively stable conformation in both solvents. The representative structures of the $\mathrm{C}[6]$ in DMSO and water resemble parallelogram, three adjacent

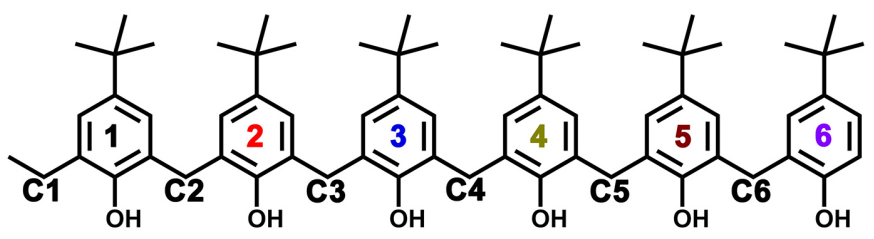

(A)

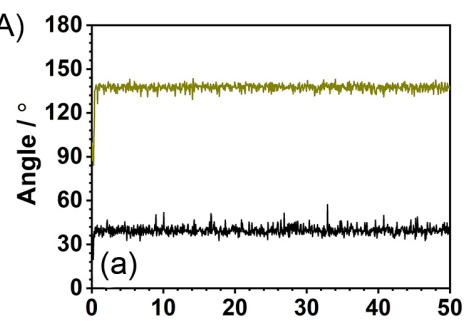

(B)

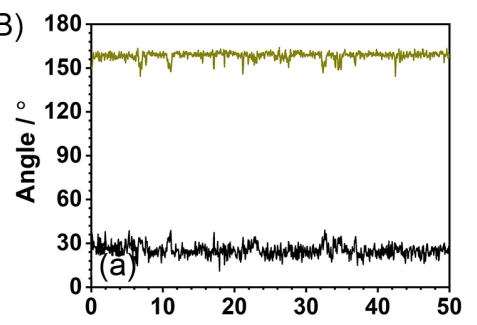

(C)

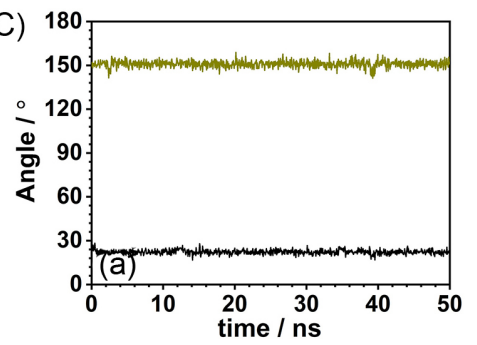

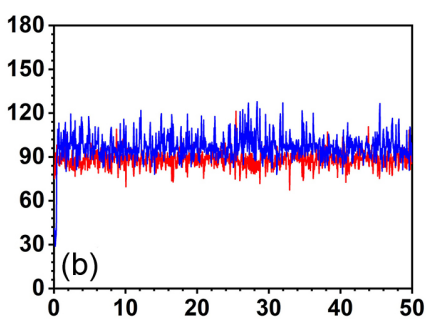
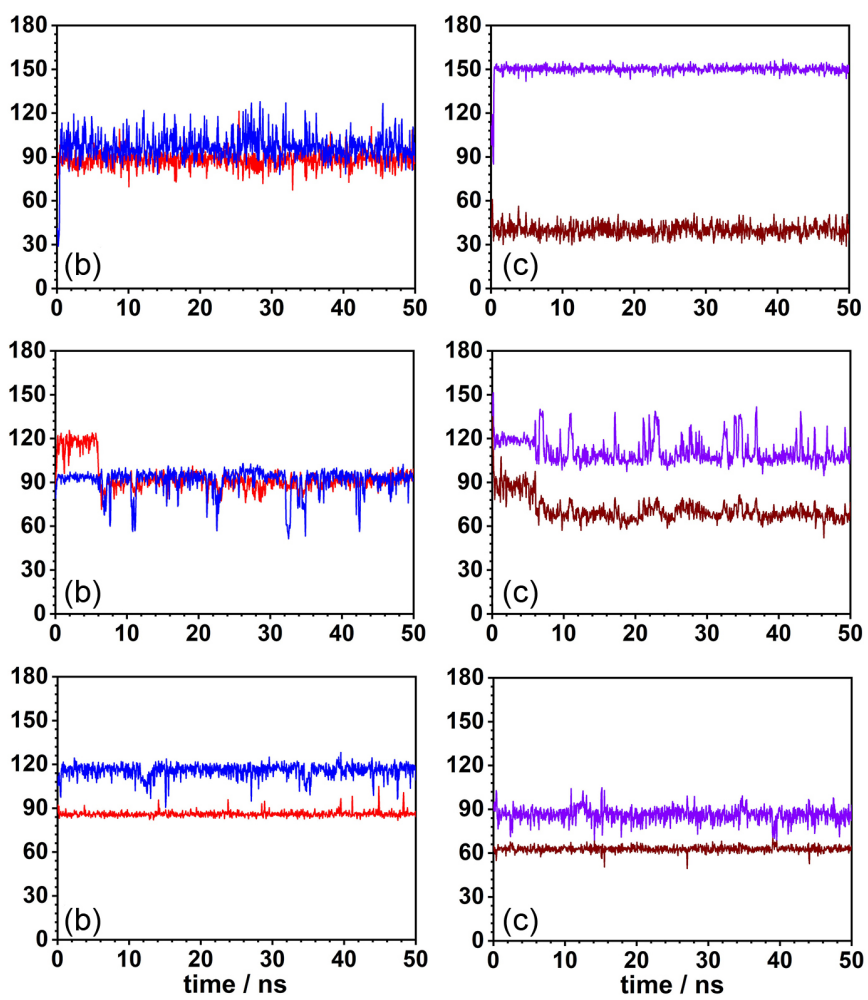

Figure 4. Evolution of the angles between the six benzene rings and the plane composed of the six methylene carbon atoms (C1, C2, C3, C4, C5, C6) along with the time in (A) $\mathrm{CHCl}_{3}$, (B) DMSO and (C) water. 


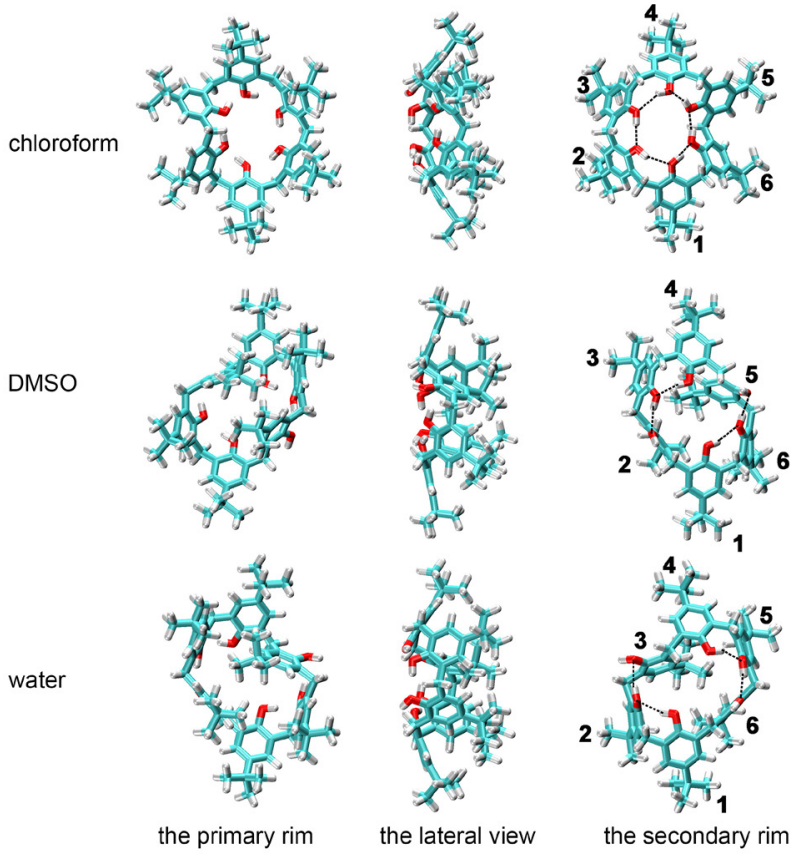

Figure 5. The primary rim, the lateral view and the secondary rim of the representative structures of the $\mathrm{C}[6]$ in three solvents, respectively. The rings $(1,2,3,4,5,6)$ correspond to those in Figure 4.

hydroxyl groups of the secondary rim form H-bonds one by one (see Figure 5).
As is known to all, $\mathrm{CHCl}_{3}$ hardly forms $\mathrm{H}$-bonds, while DMSO or water has well ability of forming H-bonds. Moreover, the $\mathrm{C}[6]$ can form not only intramolecular $\mathrm{H}$-bonds, but also intermolecular H-bonds with the DMSO or water (see "Analysis of the H-bonding interactions" sub-section). Therefore, the $\mathrm{C}[6]$ can keep a stable conformation resembling to parallelogram in DMSO and water, while it adopts an optimal conformation resembling to a chipped bowl for forming as many intramolecular $\mathrm{H}$-bonds as possible.

\section{The BA ring}

The BA ring is composed of phenyl groups and amide groups. It is reported that the structure is flexible and can undergo conformational transition in some cases. ${ }^{17,18}$ The evolution of the angles between the two opposite benzene rings along with the time is shown in Figure 6. The rings $(1,2)$ can flip around the axis of the two carbon atoms of methylene groups. In the Figure 6Aa, the angles have several abrupt turns, which indicate that benzene rings undergo a $180^{\circ}$ spin. According to Figures $6 \mathrm{Aa}-6 \mathrm{Ca}$, the abrupt turns increase gradually, illustrating that the flipped velocities are different in the three solvents, which are from low to high in order $\mathrm{CHCl}_{3}$, DMSO, water. Therefore, it can be inferred that the flipped speed of the rings $(1,2)$ of the BA ring is
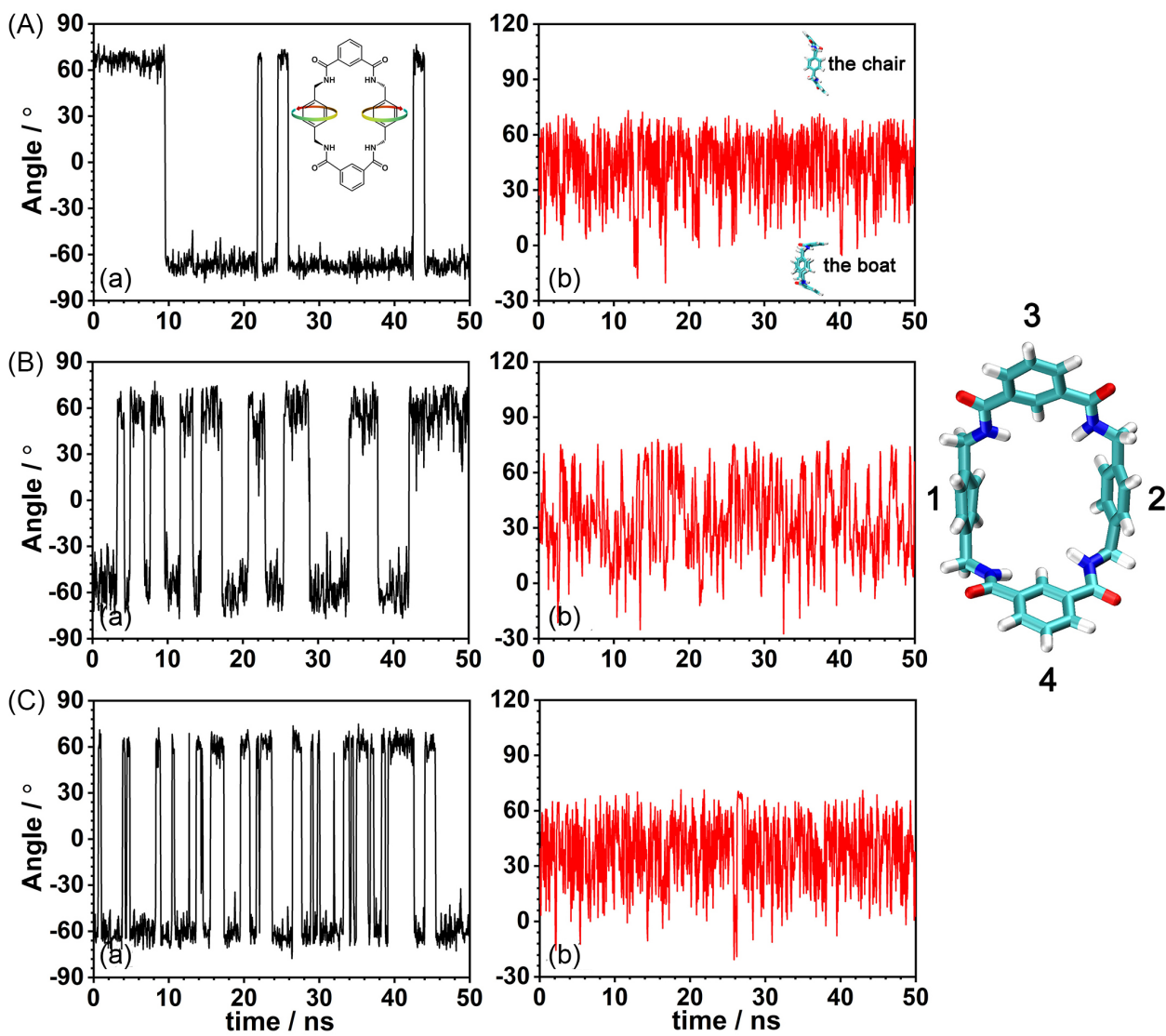

Figure 6. Evolution of the angles between the two opposite benzene rings ((a) for 1 and 2, (b) for 3 and 4 ) of the BA ring along with the increasing simulation time in (A) $\mathrm{CHCl}_{3}$, (B) DMSO and (C) water. 
related to the polarity of the solvents. The higher polarity the solvent has, the faster flipped speed the rings $(1,2)$ have. Similar to that of the $\mathrm{P}[5]$, the main reason could be that the higher polarity of the solvent led to the stronger electrostatic interactions of $\mathrm{N}$ atoms with the solvent, and thus brought about varying degrees of the flipped speed of the rings.

It can be seen in Figures $6 \mathrm{Ab}-6 \mathrm{Cb}$, the rings $(3,4)$ can swing fast from side to side. Therefore, the conformations of the BA ring can switch rapidly from the boat to the chair structure in the three solvents. It could be the flexibility of the alkyl chains on both sides of the rings $(3,4)$ that makes the rings oscillated easily. The representative structures of the BA ring are shown in Figure 7. Each structure represents a series of analogous conformations. It can be seen that the BA ring undergoes a series of conformational transformations from the boat to the chair structures, but whatever the conformations are, the structures of the BA ring are basically symmetrical.

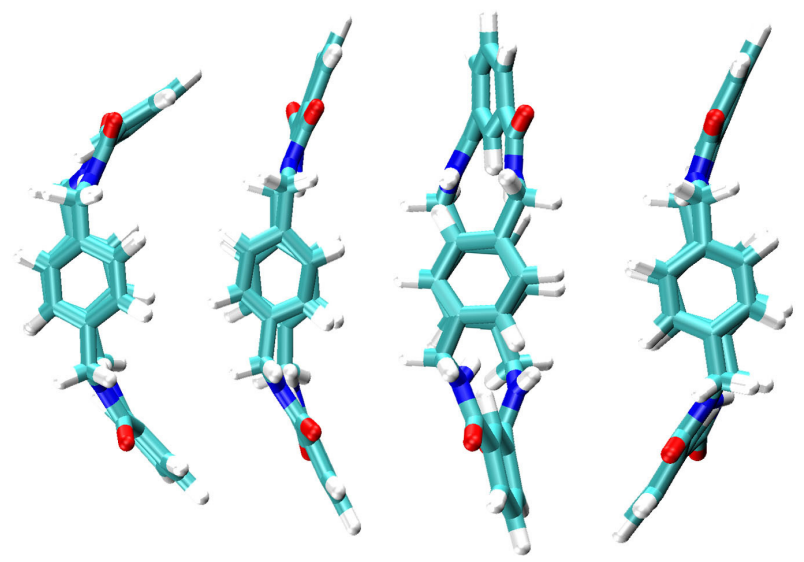

Figure 7. The lateral view of some representative structures of the BA ring.

\section{The D18C6}

The two benzene rings of the D18C6 are linked on the ortho-position with ethoxyl groups. As we know, owing to multiple $\mathrm{sp}^{3}$ carbon atoms, the D18C6 is the most flexible among the four macrocyclic molecules. For surveying the conformations of the D18C6, the evolution of the areas of the surface made up of the four carbon atoms and the distances between the two benzene rings along with the time is shown in Figure 8.

As shown in Figures $8 \mathrm{Aa}-8 \mathrm{Ca}$, the fluctuating ranges of the areas are from 12 to $28 \AA^{2}$ and there is a big difference in the three solvents. In $\mathrm{CHCl}_{3}$, the area is no more than $24 \AA^{2}$ and the profile has not a flat region. It indicates that the D18C6 tends to form folding structures and more changeable in $\mathrm{CHCl}_{3}$. Some representative structures of the D18C6 are shown in Figure 9. Similar to the BA ring, each structure represents a series of analogous conformations. By analyzing the trajectories, it is found that the D18C6 mainly adopt the folding conformations as shown in Figures $9 \mathrm{~b}-9 \mathrm{~d}$ in $\mathrm{CHCl}_{3}$. In DMSO, the fluctuating range of the areas is the greatest from 12 to $28 \AA^{2}$, and the profile exhibits some relatively flat regions. The structure at the flat regions is as shown in Figure 9a according to the trajectories. The conformation maximizes the size of the cavity. Thus, the D18C6 can form the most possible conformations from the most extensional one to the most folding one in DMSO. Instead, the area is no less than $20 \AA^{2}$ in water, and the profile exhibits more relatively flat regions, which illustrates that the D18C6 is inclined to form extensional conformations. Further, the distance between the two carbon atoms (C5 and C6) is examined for studying the pendulation of the two benzene rings. In Figures $8 \mathrm{Ab}-8 \mathrm{Cb}$, the range of distance change is the widest and most volatile from 5 to 8 in $\mathrm{CHCl}_{3}$, on the contrary, it is the narrowest and least volatile from 6.5 to 7.5 in water. That is to say that the higher polarity of the solvent results in the lesser fluctuations of the structure. The pendulation of the two benzene rings is correlative with the areas of the cavity of the D18C6, and these results reflect that the D18C6 is inclined to form extended ones in water while form folding conformations and be more changeable in $\mathrm{CHCl}_{3}$.

For investigating the level of the difficulty of transformation among conformations, the free-energy profiles characterizing the conformational stability of the D18C6 in three solvents have been obtained in Figure 10. Detail of the methodology is available in the "Free-energy calculation" sub-section of the SI section. It can be seen that the free energy of the conformations with an angle from 70 to $175^{\circ}$ are less than $5 \mathrm{kcal} \mathrm{mol}^{-1}$ in the three solvents, and in DMSO and $\mathrm{CHCl}_{3}$, the free energy is lower than that in water. It indicates that transformation among conformations in DMSO and $\mathrm{CHCl}_{3}$ are easier than in water and the structural fluctuations of the former are greater. Furthermore, in water, there is an energy valley at about $150^{\circ}$, which indicates that the conformation is the most stable state. The observations of the free-energy profiles furtherly verify the results shown in Figure 10.

Based on the above observations, it can be inferred that the conformations of the D18C6 in solvent could be related to the electrostatic interaction of $\mathrm{O}$ atoms of the poly-ethoxy chains with the solvent, which is also similar to that of the $\mathrm{P}[5]$. The higher polarity of the solvent led to the stronger electrostatic interactions of $\mathrm{O}$ atoms with the solvent, and $\mathrm{O}$ atoms prefer to be exposed to polar solvents for forming extended conformations.

On balance, the polarity of the solvent has an important influence on the macrocyclic molecules, especially these with large electronegative atoms, such as $\mathrm{O}, \mathrm{N}$. The higher polarity of the solvent led to the stronger electrostatic interactions of $\mathrm{O}$ or $\mathrm{N}$ atoms with the solvent, and thus 

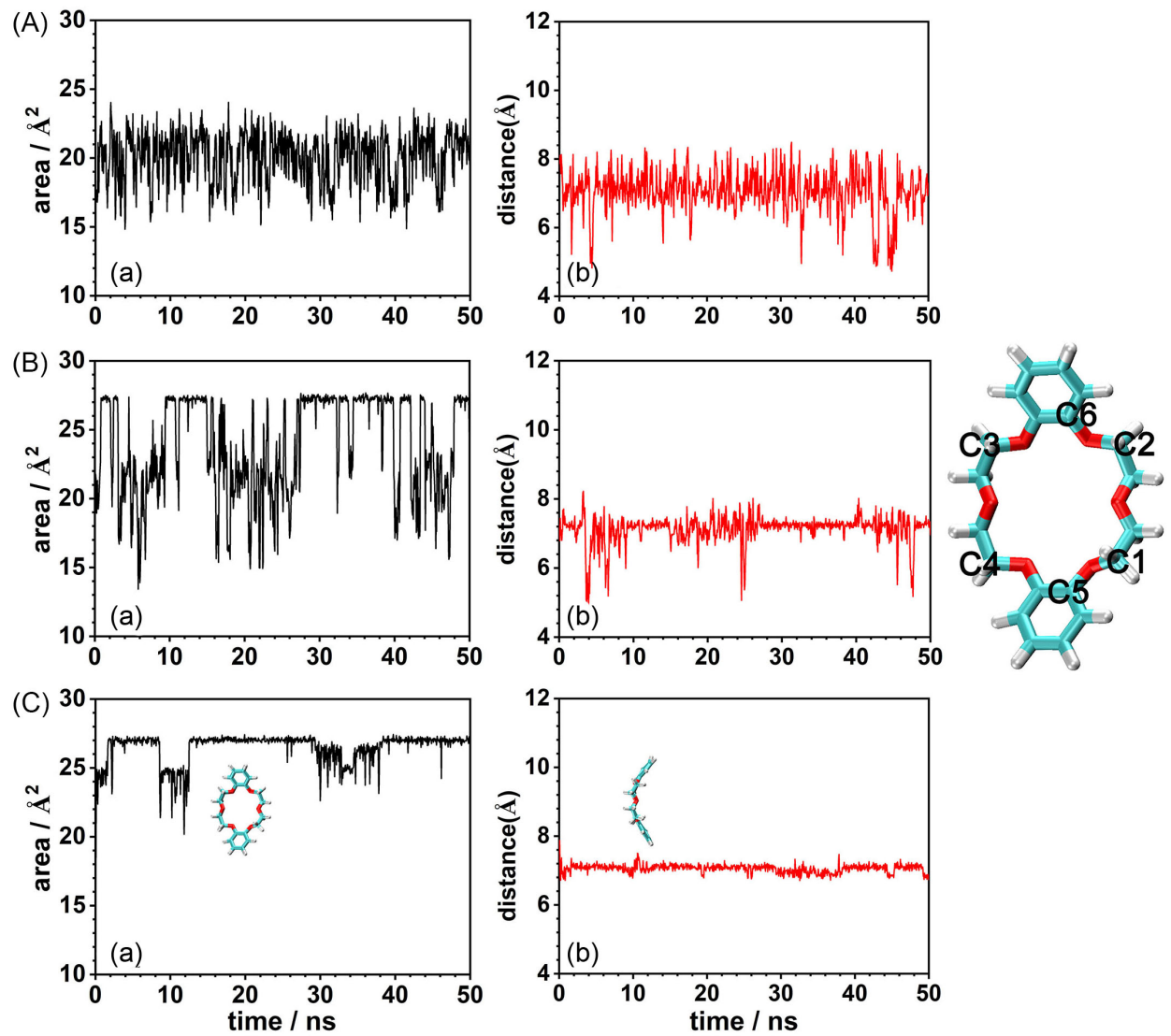

Figure 8. Evolution of (a) the areas of the surface made up of the four carbon atoms $(\mathrm{C} 1, \mathrm{C} 2, \mathrm{C} 3$ and $\mathrm{C} 4)$ and (b) the distances between the two carbon atoms (C5 and C6) along with time in (A) $\mathrm{CHCl}_{3}$, (B) DMSO and (C) water.

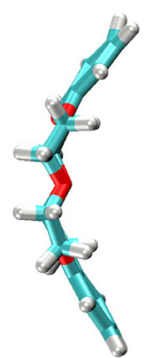

(a)

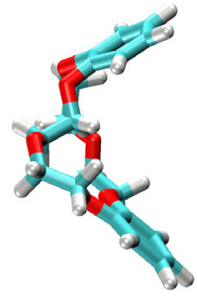

(b)

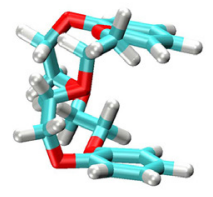

(c)

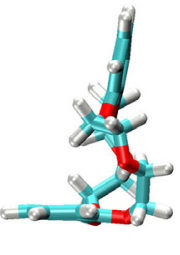

(d)

Figure 9. The flank of some representative structures of the D18C6.

these atoms prefer to be exposed to polar solvents. Therefore, for the P[5], the BA ring and the D18C6, the polarity of the solvent is the main influencing factors for their conformations. However, for the $\mathrm{C}[6]$, the polarity of the solvent has little effect on the conformations. It is the H-bonds that are the main influencing factors for the conformations. Then, for the other macrocyclic molecules besides the $\mathrm{C}[6]$, what about the $\mathrm{H}$-bonds?

\section{Analysis of the $\mathrm{H}$-bonding interactions}

As the discussion above, $\mathrm{CHCl}_{3}$ cannot form $\mathrm{H}$-bonds, while DMSO and water bears an acceptor $\mathrm{O}$ atom and can

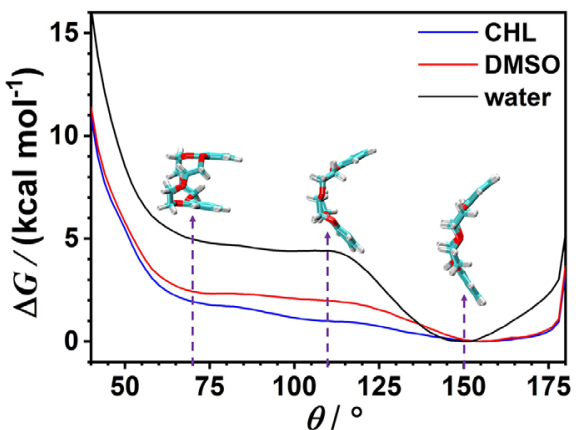

Figure 10. Free-energy profiles characterizing the conformational stability of the D18C6 along the angle $(\theta)$ formed by the three points (the centroid of the plane of the upper benzene ring; the center point of the centroid of connecting the two $\mathrm{O}$ atoms in the middle of poly (ethylene glycol) chains; the centroid of the lower benzene ring) in three solvents. The sketch map of defining $\theta$ is shown in Figure $\mathrm{S} 1$ in SI section.

form $\mathrm{H}$-bonds $(\mathrm{C}-\mathrm{H} \cdots \mathrm{O})$ with the $\mathrm{C}-\mathrm{H}$. Wherein, water can also act as H-bond donors. Among the macrocyclic molecules, the $\mathrm{P}[5]$ and the $\mathrm{C}[6]$ have hydroxyl groups, and the BA ring has amino groups, which can possess the ability of forming $\mathrm{H}$-bonding interactions (see Scheme 2). To analyze the effect of the H-bonds on the structures, the evolution of the average number of the intramolecular $\mathrm{H}$-bonds of the macrocycles and intermolecular H-bonds formed between the macrocycles and the solvents is 

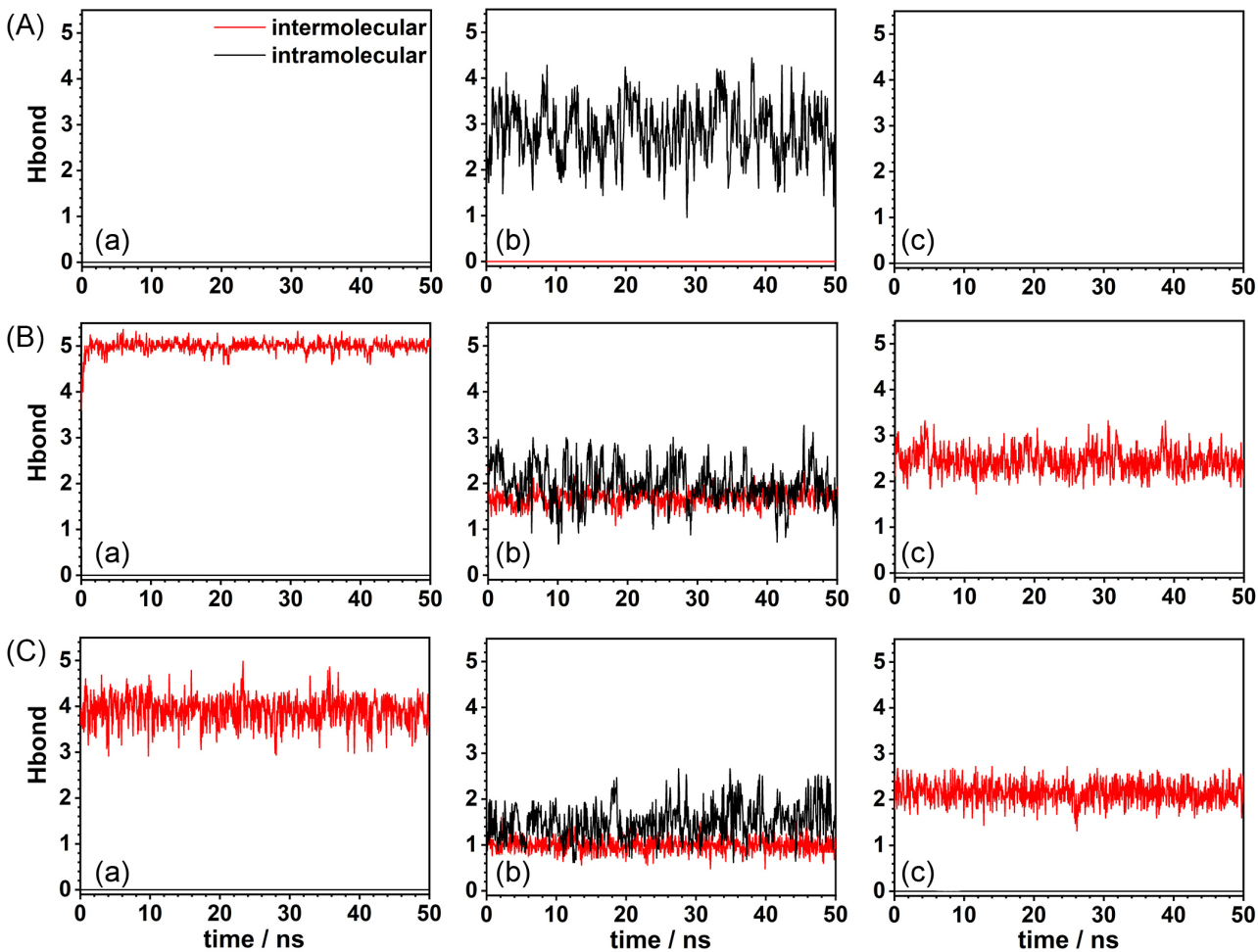

Figure 11. Evolution of the average number of intramolecular H-bonds (black line) of the macrocycles ((a) P[5], (b) C[6], (c) BA ring) and intermolecular $\mathrm{H}$-bonds (red line) formed between the macrocycles and the solvents (A) $\mathrm{CHCl}_{3}$, (B) DMSO, (C) water.

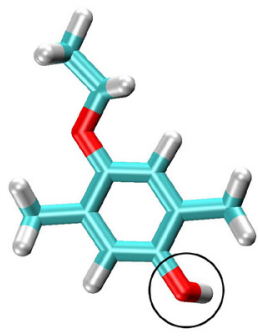

(a)

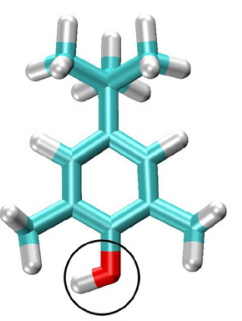

(b)

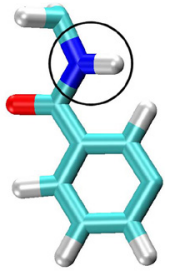

(c)
Scheme 2. Schematic illustration of a unit of the macrocyclic molecules ((a), (b), (c) for the $\mathrm{P}[5]$, the $\mathrm{C}[6]$, the BA ring, respectively). The groups circled by black circles can form $\mathrm{H}$-bonds. Wherein, $\mathrm{O}$ and $\mathrm{N}$ atom act as $\mathrm{H}$-bond acceptors and $\mathrm{O}-\mathrm{H}$ and $\mathrm{N}-\mathrm{H}$ act as $\mathrm{H}$-bond donors.

calculated by analyzing the trajectories and shown in Figure 11.

In Figure 11, the three macrocycles can form H-bonding interactions with the DMSO and water, and only the $C[6]$ can form intramolecular $\mathrm{H}$-bonds. By comparing the intermolecular H-bonds in DMSO and water, by and large, the average number of the intermolecular $\mathrm{H}$-bonds in DMSO is a little more than that in water. Furthermore, for the $\mathrm{C}[6]$, since $\mathrm{CHCl}_{3}$ cannot form $\mathrm{H}$-bonds, the macrocycles form more intramolecular $\mathrm{H}$-bonds in $\mathrm{CHCl}_{3}$ than in DMSO and water, which causes that the most stable structure in $\mathrm{CHCl}_{3}$ differs from that in DMSO and water. To summarize, the polarity of the solvents and the capability of forming intramolecular $\mathrm{H}$-bonds of the macrocyclic molecules together affect the conformations of the macrocycles.

\section{Conclusions}

In this contribution, the conformations of several macrocyclic molecules were studied in three solvents by MD simulations. The analysis of the structures of the macrocycles shows that the polarity of the solvent and the intramolecular H-bonds of the macrocycles are the main influencing factors on the conformations. For the $\mathrm{P}[5]$ and the BA ring, the solvent with higher polarity can trigger bigger structural fluctuations of the macrocyclic molecules. For the D18C6, its structure prefers to form more extensional conformations in the solvent with higher polarity. For the $\mathrm{C}[6]$, which can form intramolecular $\mathrm{H}$-bonds, the polarity of the solvent has inappreciable effect on its structure and yet both the intramolecular and intermolecular H-bonds are the main influencing factors for the structure. The present studies provide not only an understanding to the relationship between the flexibility and stability of the structures but also the effect of the solvent on the macrocyclic structures. It is also helpful for the efficient design of new macrocyclic molecules.

\section{Supplementary Information}

The supplementary information (the charge distributions, which were used for the all-atom MD simulations, of the fragments of the four macrocyclic molecules 
and the solvent molecules (water, $\mathrm{CHCl}_{3}$ and DMSO) were all provided; the methodology of the free-energy calculation characterizing the conformational stability of the D18C6 is detailed) is available free of charge at http://jbcs.sbq.org.br as PDF file.

\section{Acknowledgments}

This study is supported by Natural Science Foundation of Guangxi Province (No. 2018JJB160116), the Middleaged and Young Teachers' Basic Ability Promotion Project of Guangxi (No. 2018KY0615), Beibu Gulf University Scientific Research Foundation for the Introduction of Talent (No. 2017KYQD63) and National Natural Science Foundation of China (No. 22068001).

\section{References}

1. Pedersen, C. J.; J. Am. Chem. Soc. 1967, 89, 2495.

2. Pedersen, C. J.; J. Am. Chem. Soc. 1967, 89, 7017.

3. Song, N.; Kakuta, T.; Yamagishi, T.; Yang, Y. W.; Ogoshi, T.; Chem 2018, 4, 2029.

4. Ovsyannikov, A.; Solovieva, S.; Antipin, I.; Ferlay, S.; Coord. Chem. Rev. 2017, 352, 151.

5. Ogoshi, T.; Kanai, S.; Fujinami, S.; Yamagishi, T.; Nakamoto, Y.; J. Am. Chem. Soc. 2008, 130, 5022.

6. Xue, M.; Yang, Y.; Chi, X. D.; Zhang, Z. B.; Huang, F. H.; Acc. Chem. Res. 2012, 45, 1294.

7. Zhou, Y. J.; Jie, K. C.; Zhao, R.; Li, E.; Huang, F. H.; J. Am. Chem. Soc. 2020, 142, 6957.

8. Leigh, D. A.; Murphy, A.; Smart, J. P.; Deleuze, M. S.; Zerbetto, F.; J. Am. Chem. Soc. 1998, 120, 6458.

9. Gatti, F. G.; León, S.; Wong, J. K. Y.; Bottari, G.; Altieri, A.; Morales, M. A. F.; Teat, S. J.; Frochot, C.; Leigh, D. A.; Brouwer, A. M.; Zerbetto, F.; Proc. Natl. Acad. Sci. U. S. A. 2003, 100, 10 .

10. Liu, Z. C.; Nalluri, S.; Stoddart, J. F.; Chem. Soc. Rev. 2017, 46, 2459.

11. Xue, M.; Yang, Y.; Chi, X. D.; Yan, X. Z.; Huang, F. H.; Chem. Rev. 2015, 115, 7398 .

12. Bruns, C. J.; Stoddart, J. F.; Acc. Chem. Res. 2014, 47, 2186.

13. Mewis, R. E.; Archibald, S. J.; Chem. Rev. 2010, 254, 1686.

14. Mallinson, J.; Collins, I.; Future Med. Chem. 2012, 4, 1409.

15. Marsault, E.; Peterson, M. L.; J. Med. Chem. 2011, 54, 1961.

16. Reinhoudt, D. N. In Calixarenes and Beyond; Neri, P.; Sessler, J. L.; Wang, M.-X., eds.; Springer: Cham, Switzerland, 2016, p. 3.
17. Boulet, B.; Joubert, L.; Cote, G.; Bouvier-Capely, C.; Cossonnet, C.; Adamo, C.; J. Phys. Chem. A 2006, 110, 5782.

18. Panman, M. R.; Bakker, B. H.; den Uyl, D.; Kay, E. R.; Leigh, D. A.; Buma, W. J.; Brouwer, A. M.; Geenevasen, J. A.; Woutersen, S.; Nat. Chem. 2013, 5, 929.

19. Liu, P.; Shao, X. G.; Chipot, C.; Cai, W. S.; Chem. Sci. 2016, $7,457$.

20. Fu, H. H.; Shao, X. G.; Chipot, C.; Chem. Sci. 2017, 8, 5087.

21. Bushuev, Y. G.; Usacheva, T. R.; Sharnin, V. A.; J. Mol. Liq. 2016, 224, 825.

22. Olschewski, M.; Knop, S.; Seehusen, J.; Lindner, J.; Vöhringer, P.; J. Phys. Chem. A 2011, 115, 1210.

23. Gutsche, C. D.; The Calixarenes; Springer: Berlin, Heidelberg, 1984.

24. Johnston, A. G.; Leigh, D. A.; Pritchard, R. J.; Deegan, M. D.; Angew. Chem., Int. Ed. Engl. 1995, 34, 1209.

25. Patajczak, H. M.; Lis, T.; Rusek, G.; J. Mol. Struct. 1994, 327, 241.

26. Humphrey, W.; Dalke, A.; Schulten, K.; J. Mol. Graphics 1996, $14,33$.

27. Feller, S. E.; Zhang, Y. H.; Pastor, R. W.; Brooks, B. R.; J. Chem. Phys. 1995, 103, 4613.

28. Phillips, J. C.; Braun, R.; Wang, W.; Gumbart, J.; Tajkhorshid, E.; Villa, E.; Chipot, C.; Skeel, R. D.; Kalé, L.; Schulten, K.; J. Comput. Chem. 2005, 26, 1781.

29. Vanommeslaeghe, K.; Hatcher, E.; Acharya, C.; Kundu, S.; Zhong, S.; Shim, J.; Darian, E.; Guvench, O.; Lopes, P.; Vorobyov, I.; Mackerell Jr., A. D.; J. Comput. Chem. 2010, 31, 671.

30. Jorgensen, W. L.; Chandrasekhar, J.; Madura, J. D.; Impey, R. W.; Klein, M. L.; J. Chem. Phys. 1983, 79, 926.

31. Dietz, W.; Heinzinger, K.; Ber. Bunsen. Phys. Chem. 1985, 89, 968.

32. Strader, M. L.; Feller, S. E.; J. Phys. Chem. A 2002, 106, 1074.

33. Ryckaert, J. P.; Ciccotti, G.; Berendsen, H. J. C.; J. Comput. Phys. 1977, 23, 327.

34. Andersen, H. C.; J. Comput. Phys. 1983, 52, 24.

35. Tuckerman, M.; Berne, B. J.; Martyna, G. J.; J. Chem. Phys. 1992, 97, 1990.

36. Darden, T.; York, D.; Pedersen, L.; J. Chem. Phys. 1993, 98, 10089.
Submitted: April 17, 2021

Published online: June 15, 2021 